\title{
Reproductive phenology of useful Seasonally Dry Tropical Forest trees: Guiding patterns for seed collection and plant propagation in nurseries
}

\author{
Adriana L. Luna-Nieves ${ }^{a}$, Jorge A. Meave ${ }^{b}$, Leonor Patrícia Cerdeira Morellato ${ }^{c}$, \\ Guillermo Ibarra-Manríquez ${ }^{\mathrm{a}, *}$
}

${ }^{a}$ Instituto de Investigaciones en Ecosistemas y Sustentabilidad, Universidad Nacional Autónoma de México, Antigua Carretera a Pátzcuaro 8701, Col. Ex Hacienda de San José de La Huerta, C.P. 58190 Morelia, Michoacán, Mexico

${ }^{\mathrm{b}}$ Departamento de Ecología y Recursos Naturales, Facultad de Ciencias, Universidad Nacional Autónoma de México, Ciudad de México 04510, Mexico

${ }^{\mathrm{c}}$ Universidade Estadual Paulista (UNESP), Instituto de Biociências, Departamento de Botânica, Laboratório de Fenologia, CEP 13506-900 Rio Claro, São Paulo, Brazil

\section{A R T I C L E I N F O}

\section{Article history:}

Received 10 November 2016

Received in revised form 4 March 2017

Accepted 10 March 2017

Available online 23 March 2017

\section{Keywords:}

Adaptive management

Community-based monitoring program

Environmental trigger

Flowering time

Fruiting time

Seed collection

\begin{abstract}
A B S T R A C T
The propagation in nurseries of native plant species potentially useful for agroforestry, silvopastoral and restoration programs is hindered by an inadequate supply of high quality seed. Limitations in our knowledge on the phenological patterns of native species result in the lack of efficient collecting protocols. Here we analyze the reproductive phenology of 14 native tree species from Seasonally Dry Tropical Forest (SDTF) that are widely used in reforestation and restoration programs. We conducted monthly observations during five years through a community-based monitoring program in two conservation areas within the Zicuirán-Infiernillo Biosphere Reserve (West Mexico) to assess the flowering and fruiting phenology of 149 marked trees (7-20 trees per species). For each species we described the phenophase intensity, duration, seasonality, synchrony and frequency. We related the intensity of reproductive phenology to climatic variables (photoperiod, precipitation and temperature). We identified three main phenological strategies of SDTF species that differ in timing and climatic triggers: (1) flowering and fruiting exclusively in the rainy season; (2) flowering in the rainy season and fruiting in the dry season; and (3) flowering and fruiting exclusively in the dry season. For each phenological strategy we make recommendations of optimal collecting seeds schedules. The community-based monitoring program, which involves the participation of local social actors, guaranteed the success of long-term phenological monitoring. Our study provides valuable information on both the inter-annual and inter-specific variation of the phenological patterns of tree species of forestry interest, and demonstrates that qualitative descriptions of population-level phenological attributes is an essential input to develop adaptive management programs.
\end{abstract}

(c) 2017 Elsevier B.V. All rights reserved.

\section{Introduction}

A major hindrance faced by nurseries devoted to the propagation of native plant species used in agroforestry, silvopastoral or restoration programs, is the lack of a timely and permanent supply of high-quality seed (Böhringer et al., 2003; Botha et al., 2005, 2006). This situation reflects deficiencies in our knowledge on the reproductive biology of native species, in particular, their phenological patterns and the optimal times for plant reproduction when the best seeds are produced. Ultimately, the deficiency of phenological data results in the lack of efficient collecting protocols

\footnotetext{
* Corresponding author.

E-mail addresses: aluna@iies.unam.mx (A.L. Luna-Nieves), jorge.meave@ ciencias.unam.mx (J.A. Meave), pmorella@rc.unesp.br (L.P.C. Morellato), gibarra@
} cieco.unam.mx (G. Ibarra-Manríquez).
(Buisson et al., 2016; Fenner and Thompson, 2004; Kuaraksa et al., 2012).

Phenological studies seek to describe the timing of cyclic biological events and to identify their internal and external clues. In a plant's life cycle a distinction is made between two major reproductive phenophases, namely flowering and fruiting. The importance of accurate descriptions of reproductive phenological patterns for the design of conservation or management strategies and ecological restoration programs has recently been recognized (Morellato et al., 2016). The relevance relies in the need for phenological information to recognize specific times of the year with high flower, fruit and seed availability, to assess the duration, intensity, frequency and synchrony of their occurrence during the year, and to evaluate differences in phenological patterns between species, populations and communities (Miller-Rushing and Weltzin, 2009; Newstrom et al., 1994). 
In the context of Seasonally Dry Tropical Forests (SDTF), phenological information is of particular interest, since compared with temperate and tropical wet forest ecosystems, the diverse and complex phenological patterns of SDTF are still poorly explored and little is known for native species with forestry potential (Bonfil and Trejo, 2010). Most phenological studies conducted in SDTF have focused on the description of community-level patterns (Bullock and Solís-Magallanes, 1990; Cortés-Flores et al., 2017; Frankie et al., 1974; Justiniano and Fredericksen, 2000), while the assessment of such patterns at the population level has received less attention (Kuaraksa et al., 2012; Venter and Witkowski, 2011; Wallace and Painter, 2002).

The main drivers of the overall phenological patterns displayed by the SDTF tree community are precipitation seasonality and intensity, as the occurrence of the growth and reproduction largely depends on the variation of water availability throughout the year or the length of rainy season (Borchert, 1998; Borchert et al., 2002, 2004; Holbrook et al., 1995; McLaren and McDonald, 2005; Morellato et al., 2013). Therefore, in ecosystems with a marked dry season that lasts between four and eight months, phenological strategies have reflected the range of adaptations allowing plants to tolerate and survive the seasonal drought (Borchert et al., 2004; Morellato et al., 2013; Singh and Kushwaha, 2006). With respect to flowering, two major strategies have been identified (Singh and Kushwaha, 2006): species in which flower buds develop in response to increasing water availability during the rainy season, and species that flower in the dry season, either at the beginning (just after the end of the rainy season, in the fall), or at the end (several weeks before the onset of the rains, in the spring). For this latter group of species, changes in the photoperiod apparently trigger reproductive events in plants. According to Singh and Kushwaha (2005), the drier is a seasonal tropical forest, the larger is the number of species flowering during the dry season. In turn, fruit maturation concentrates almost exclusively in the dry period of the year, which is the season when seed dispersal by wind is favored, with the exception of fleshy fruits, which tend to mature in the rainy season, when environmental conditions favor maturation and seed dispersers are abundant (Bullock and SolísMagallanes, 1990; Frankie et al., 1974; Justiniano and Fredericksen, 2000; Morellato et al., 2013; Singh and Kushwaha, 2005). Regardless of the timing of seed dispersal, most seeds of SDTF trees remain dormant until the beginning of the rainy season, when they germinate, an evolutionarily selected trait that may increase the probability for successful seedling establishment (Frankie et al., 1974; van Schaik et al., 1993).

Despite the generality of these findings, community phenological patterns may conceal a large diversity of phenological responses displayed by the tree species, which only emerges when patterns are assessed at the population level. When taking this approach, it becomes evident that plants' responses to changing climatic conditions throughout the year are no longer predictable, given the plethora of strategies that plants have evolved to face seasonal water limitations (Borchert et al., 2002; Singh and Kushwaha, 2005). Within this framework, we proposed the use of a community-based monitoring program involving the local inhabitants to conduct a five-year long phenology observation. We aimed to investigate the reproductive phenological patterns of STDF tree species with forestry potential, in order to provide essential information for the planning of seed collection schedules and their subsequent propagation in nurseries, while focusing on ecological restoration. We also evaluated the influence of climatic conditions on the onset of flowering and fruiting, and examined whether these STDF tree species differ in their reproductive strategies, reflecting divergent evolutionary responses to the dry season.

We hypothesized that in this highly seasonal environment, tree phenology responds primarily to rainfall seasonality, as this is the main constraining factor; consequently, inter-annual phenological variation should be mainly driven by rainfall variability. Alternatively, we hypothesized that phenological timing of species responds mainly to factors that are largely invariable among years, such as photoperiod, which would result in high predictability of the phenological patterns, regardless the inter-annual rainfall variation. If the amount of inter-annual variation (precipitation) overrides the regularity of within-year seasonal variation (photoperiod), this would result in greater phenological uncertainty among years. Clearly, in the context of a nursery-based plant propagation program it is important to discriminate between these contrasting hypotheses.

\section{Materials and methods}

\subsection{Study site}

The study was conducted in the lower portion of the Balsas river basin, Churumuco municipio (county), Michoacán state, Western Mexico (extreme coordinates $18^{\circ} 38^{\prime}-18^{\circ} 44^{\prime} \mathrm{N}, 101^{\circ} 38^{\prime}-101^{\circ} 41^{\prime}$ $\mathrm{W})$. The area is part of the buffer zone of the Zicuirán-Infiernillo Biosphere Reserve and comprises of 892 ha, with an elevation range of 300-1200 m asl.

The regional climate is tropical dry with a summer rainfall regime $\left(\mathrm{BS}_{0}\right)$, with a mean annual temperature of $28^{\circ} \mathrm{C}$ and a mean total annual precipitation of $650 \mathrm{~mm}$, strongly concentrated between June and September (Fig. 1). The prevailing vegetation type is the SDTF, characterized by a large proportion $(>75 \%)$ of trees shedding all their leaves in the dry season, and a more or less continuous canopy with an average height of $8 \mathrm{~m}$. In addition to the SDTF, in areas with higher soil moisture, such as ravines or close to water streams, the vegetation is a semi-evergreen tropical forest characterized by a smaller proportion of individuals shedding their leaves in the dry season (50-70\%), and the presence of dominant trees with $>15 \mathrm{~m}$ height.

\subsection{Species selection}

The study species were selected according to three criteria: local, governmental and conservation status. Species with local interest were identified through 16 interviews with local people, who distinguish which species were the most preferred by the inhabitants of the region for different purposes (construction, fodder, food, medicine, ornament or poles). Governmental importance was identified through 10 interviews with officials from public institutions of the forestry sector, to identify species promoted by government agencies for propagation in reforestation and restoration programs, for which reason they have a high regional trade potential. Finally, we assessed conservation relevance based on the classification of species of interest for local inhabitants and government agencies in conservation or threat categories according to the Mexican environmental legislation (SEMARNAT, 2010). From 38 species mentioned by the two groups of interviewees we selected 14 that coincided in the selection criteria (Table 1).

\subsection{Reproductive phenology}

We established a $15 \mathrm{~km}$ line transect in the study region along an elevation gradient from 200 to $1000 \mathrm{~m}$, which comprised three portions: (1) low (230-480 m, where prevailing physiography is slope foothills and prevailing vegetation is secondary SDTF), (2) medium (481-725 m, mostly medium slopes with moderate steepness, where SDTF is intermingled with old secondary stands), and (3) high (726 and 975, with SDTF and tropical semi-evergreen forest along a water stream). 


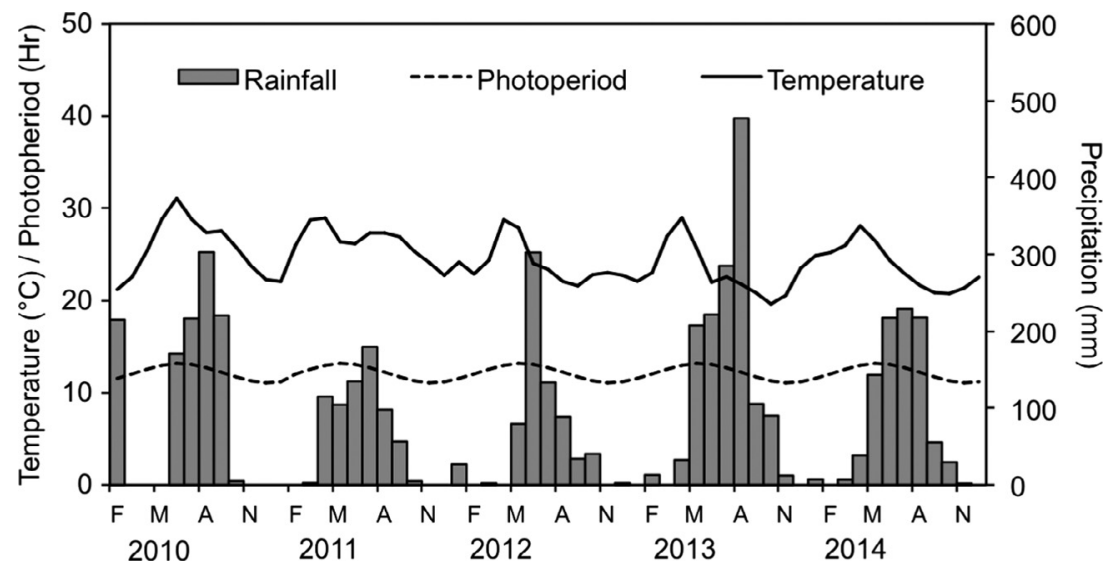

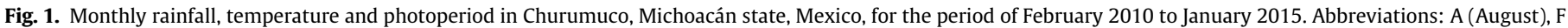
(February), M (May), N (November).

Table 1

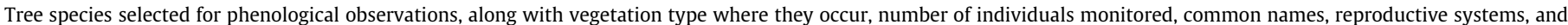

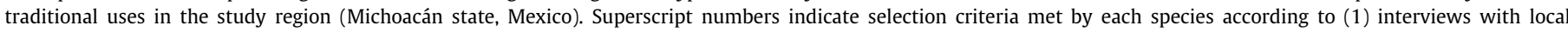

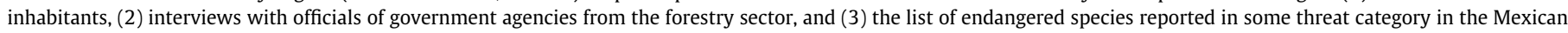
environmental law (SEMARNAT, 2010). Number of individuals observed for each species is indicated in parenthesis.

\begin{tabular}{|c|c|c|c|}
\hline Family/Species & Common name & Sexual system & Uses \\
\hline \multicolumn{4}{|l|}{ Seasonally Dry Tropical Forest } \\
\hline \multicolumn{4}{|l|}{ Anacardiaceae } \\
\hline Amphipterygium adstringens (Schltdl.) Standl., $\mathbf{1 , 2}(\mathbf{1 0})$ & Chacualalate & Dioecious & Medicinal \\
\hline \multicolumn{4}{|l|}{ Apocynaceae } \\
\hline Plumeria rubra L. ${ }^{2}(\mathbf{1 0})$ & Parandazicua & Hermaphroditic & Ornamental \\
\hline \multicolumn{4}{|l|}{ Bignoniaceae } \\
\hline Handroanthus impetiginosus (Mart. ex DC.) Mattos ${ }^{\mathbf{2 , 3}}$ (10) & Cañafístula & Hermaphroditic & Construction \\
\hline \multicolumn{4}{|l|}{ Boraginaceae } \\
\hline Cordia elaeagnoides DC. ${ }^{\mathbf{2}(\mathbf{1 0})}$ & Cuerámo & Hermaphroditic & Construction, medicinal, poles \\
\hline \multicolumn{4}{|l|}{ Leguminosae } \\
\hline Caesalpinia coriaria (Jacq.) Willd. ${ }^{\mathbf{1}(\mathbf{1 0})}$ & Cascalote & Hermaphroditic & Forage, commercial \\
\hline Caesalpinia platyloba S. Watson $\mathbf{1 , 2}(\mathbf{1 0})$ & Frijolillo & Dioecious & Construction, poles \\
\hline Eysenhardtia polystachya (Ortega) Sarg. ${ }^{\mathbf{1}(\mathbf{1 0})}$ & Palo dulce & Hermaphroditic & Medicinal, poles \\
\hline Gliricidia sepium (Jacq.) Kunth ex Walp. ${ }^{1}$ (10) & Trébol & Hermaphroditic & Poles, forage \\
\hline Heteroflorum sclerocarpum M. Sousa ${ }^{1}(\mathbf{1 0})$ & Ojo de perico & Dioecious & Forage \\
\hline \multicolumn{4}{|l|}{ Malvaceae } \\
\hline Guazuma ulmifolia Lam., (10) & Cahulote & Hermaphroditic & Forage, poles \\
\hline \multicolumn{4}{|l|}{ Semideciduous Tropical Dry Forest } \\
\hline \multicolumn{4}{|l|}{ Burseraceae } \\
\hline Bursera fragrantissima Bullock $\mathbf{1}^{\mathbf{2 0})}$ & Tecomaca & Dioecious & Medicinal \\
\hline \multicolumn{4}{|l|}{ Chrysobalanaceae } \\
\hline Licania arborea Seem. $^{2,3}$ (7) & Cuirindal & Hermaphroditic & Forage \\
\hline \multicolumn{4}{|l|}{ Meliaceae } \\
\hline Swietenia humilis Zucc. ${ }^{2}$ (7) & Cóbano & Monoecious & Furniture, medicinal \\
\hline \multicolumn{4}{|l|}{ Moraceae } \\
\hline Brosimum alicastrum Sw. ${ }^{\mathbf{1 , 2}}$ (15) & Uje & Monoecious & Forage, food \\
\hline
\end{tabular}

We characterized the distribution and abundance of the study species by recording every individual located within $50 \mathrm{~m}$ at each side of the line transect and logging their precise location with a GPS (Appendix A). Depending on their abundances, we then marked between 7 and 20 individuals of each species (149 trees in total), all of them having a minimum diameter at breast height of $10 \mathrm{~cm}$, as trees of this size or bigger have a high probability of being reproductive (Bullock and Solís-Magallanes, 1990). The selected trees were separated by distances $>50 \mathrm{~m}$ to minimize genetic relatedness and space dependency of data.

We conducted monthly observations with binoculars in the last week of each of 60 months, from February 2010 to February 2014 (with the single exception of February 2011). The percent of the crown occupied by flowers in anthesis (flowering) and mature fruits (fruiting) in each marked individual was assessed by using Fournier's (1974) semi-quantitative scale of five categories (0-4) with an interval of $25 \%$ between each one.

\subsection{Community-based phenology monitoring program}

A community monitoring team conducted the quinquennial evaluation of the reproductive phenology on which this study is based. This team emerged as a strategy aimed to motive the participation, organization and training of community members in the management of native trees species of forestry interest. During the first monitoring year, the coordinators of this project accompanied the monitoring team, which was formed for six peasants $>40$ years old. In this way, the monitoring team received training on the identification of the different development stages of the reproductive structures of the study species, and on the use of the equipment needed for the evaluations. During the four following years the fieldwork was the exclusive responsibility of the community monitoring team. To increase the reliability of the results so gathered, we conducted four workshops during the first year of study to calibrate the phenological observations. In them, 


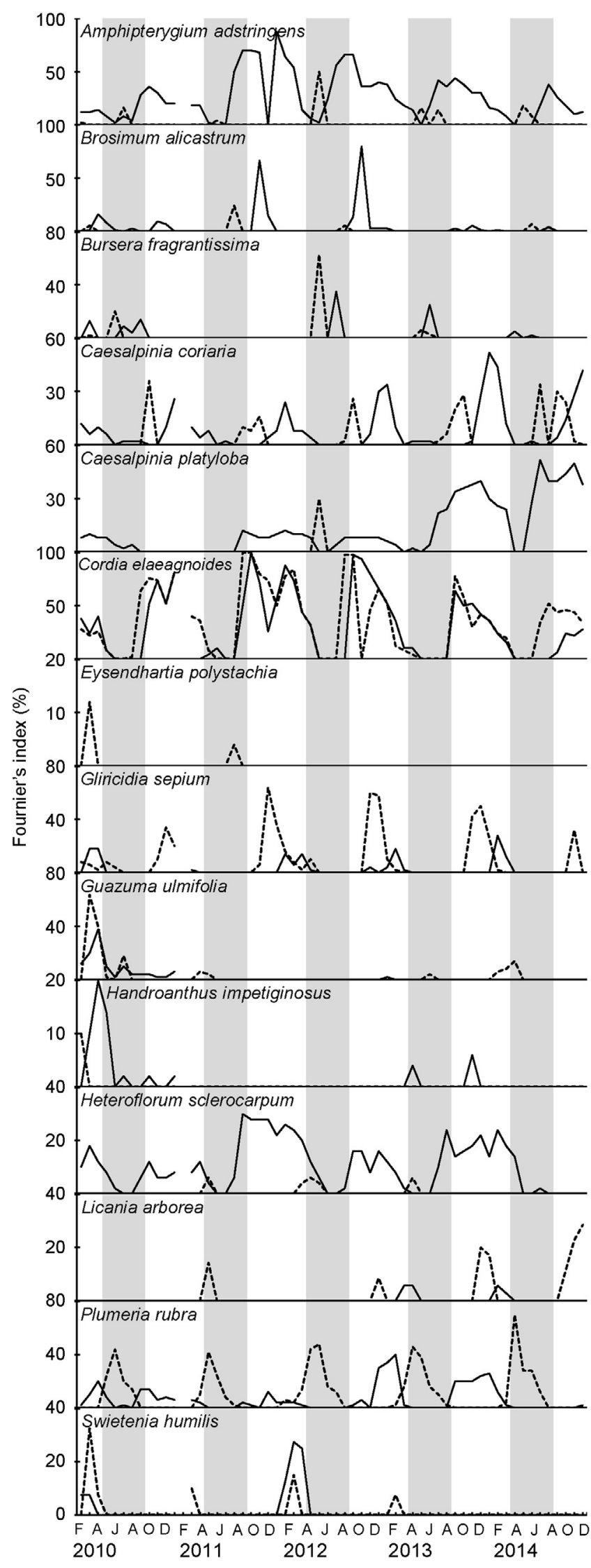

Fig. 2. Percent intensity index of flowering (dashed lines) and fruiting (solid lines) of 14 Seasonally Dry Tropical Forest tree species (Churumuco, Michoacán state, Mexico) from February 2010, to February 2015. Abbreviations: A (April or August), D (December), F (February), J (June), O (October). Gray bars indicate the rainy season. each member of the monitoring group evaluated the presence of flowers and fruits independently. Then, we discussed together likely reasons for the discrepancies. In the last two workshops, the match between the different observers was approximately $95 \%$.

\subsection{Climatic data}

We obtained daily minimum and maximum temperatures, and daily precipitation, from the closest meteorological station to our study area (National Water Commission, Las Cruces de Turicato, $19^{\circ} 3^{\prime} 55^{\prime \prime} \mathrm{N}, 101^{\circ} 25^{\prime} 40^{\prime \prime} \mathrm{W}, 800 \mathrm{~m}$ asl). Due to technical problems, we were only able to obtain climatic data for the 2010-2013 period. Information for 2014 was obtained through extrapolation, using an ARIMA model in forecast package in $\mathrm{R}$ ( $\mathrm{R}$ Core Team, 2014) and taking into consideration the historical climate records of the region (Fig. 1). Daylight duration was obtained from the Astronomical Applications Department of the U.S. Naval Observatory (http://www.usno.navy.mil).

\subsection{Data analysis}

We calculated Fournier's (1974) intensity index to evaluate the proportion of flowering and fruiting in each species and month (values < 25\% indicate a low intensity, those between 25 and $50 \%$ indicate a medium intensity; and those $>50 \%$ a high intensity). Additionally, we described the frequency of the phenophases (number of reproductive cycles by year) as annual if event occurred only once in the year, and supra-annual if the time elapsed between two phenological events was larger than one year, or if it was not consecutive over the five-year study period.

Synchrony was assessed through the index proposed by Freitas and Bolmgren (2008), which indicates whether the intensity peak of a given phenophase coincides completely (1) or not at all (0) among all individuals within a population (values $<0.25$ signify that the species had an asynchronic reproductive event, values between 0.25 and 0.5 indicate that an event had low synchrony, and those values $>0.5$ denoted a synchronic event).

Duration (the time elapsed between the start and the end of a phenophase) was described by calculating the mean and the standard deviation of the number of months by year in which the two phenophases were observed. The presence of flowers and/or fruits in at least one individual was sufficient to indicate that a given species was flowering or fruiting in that month. Based on the mean number of months in which the phenophase occurred, the duration was classified as short ( $<2$ months), intermediate (2-5 months) or extended ( $>5$ months).

To assess phenophase seasonality we used circular statistics to test the null hypothesis that the presence of flowers and fruits was homogeneously distributed around the year as proposed by Morellato et al. (2000). We assigned to each month an angular value of $30^{\circ}$ (February, $0^{\circ}$; March, $30^{\circ}$; April $60^{\circ}$; etc.), and calculated the mean angle $\alpha$ and the magnitude of the $r$ vector; $\alpha$ indicates the time (month) of the year in which the largest number of individuals of a given species presented a phenophase, while $r$ indicates the degree of dispersion or concentration of the observations (Zar, 1999). The $r$ vector values range from 0 (indicating a totally homogeneous distribution of the data around the year) to 1 (indicating all data are concentrated in a single angle or data) and can be considered as a proxy of seasonality (Morellato et al., 2000, 2010). The significance of the mean angle $\alpha$ was tested through a Rayleigh's (z) test (Zar, 1999).

Finally, we analyzed the relationship between flowering and fruiting (monthly Fournier's intensity index for each phenophase) and climatic variables (mean monthly precipitation and photoperiod) through generalized linear models (GLM) with binomial error and a logit link function; this analysis was implemented in 
Table 2

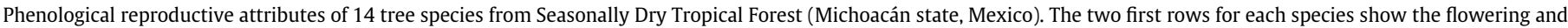

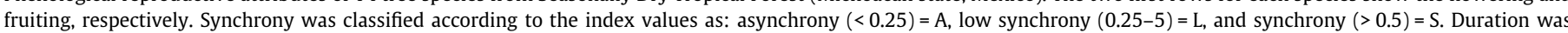

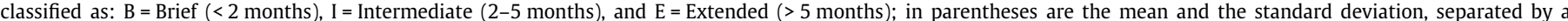

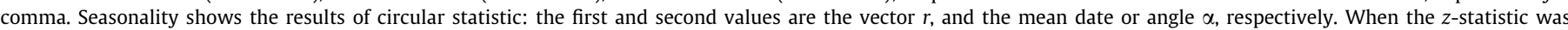
significant, $P \leq 0.05\left(^{*}\right)$, the mean date is shown in parentheses. The symbol (-) indicates that the phenophase was not observed.

\begin{tabular}{|c|c|c|c|c|c|c|}
\hline Species & Attribute & 2010 & 2011 & 2012 & 2013 & 2014 \\
\hline $\begin{array}{c}\text { Amphipterygium } \\
\text { adstringens }\end{array}$ & $\begin{array}{l}\text { Synchrony } \\
\text { Duration } \\
\text { Seasonality }\end{array}$ & $\begin{array}{l}\mathrm{L}(0.5) \\
\mathrm{A}(0.14) \\
\mathrm{B}(0.5,0.7) \\
\mathrm{E}(6,4.5) \\
0.6-140.9^{\circ}(21 \text { June }) \\
0.2-38.2^{\circ}\end{array}$ & $\begin{array}{l}\text { A }(0) \\
\text { S }(0.53) \\
B(0.1,0.3) \\
\text { E }(6,1.9) \\
1-120^{\circ}(1 \text { June) } \\
0.2-68.6^{\circ *} \text { (9 April) }\end{array}$ & $\begin{array}{l}\mathrm{L}(0.29) \\
\mathrm{L}(0.32) \\
\mathrm{B}(0.7,0.5) \\
\mathrm{E}(6.7,4.3) \\
1-120^{\circ} \\
0.2-69-6^{\circ}\end{array}$ & $\begin{array}{l}A(0.03) \\
A(0.2) \\
B(0.5,0.8) \\
E(7,4.7) \\
0.9-143.4^{\circ} \\
0.2-83.6^{\circ}\end{array}$ & $\begin{array}{l}\mathrm{A}(0.04) \\
\mathrm{A}(0.12) \\
\mathrm{B}(0.4,0.7) \\
\mathrm{I}(4,3.2) \\
1-127.4^{\circ} \\
0.3-61.1^{\circ}(1 \text { April })\end{array}$ \\
\hline Brosimum alicastrum & $\begin{array}{l}\text { Synchrony } \\
\text { Duration } \\
\text { Seasonality }\end{array}$ & $\begin{array}{l}\mathrm{A}(0.1) \\
\mathrm{A}(0) \\
\mathrm{B}(0.3,0.5) \\
\mathrm{B}(1.7,1.6) \\
0.1-30^{\circ} \\
0.1-6.2^{\circ}\end{array}$ & $\begin{array}{l}\text { A }(0.21) \\
\mathrm{L}(0.45) \\
\mathrm{B}(0.9,0.4) \\
\mathrm{B}(1.5,0.5) \\
1-180^{\circ} \\
1-79.7^{\circ *}(20 \text { April })\end{array}$ & $\begin{array}{l}\mathrm{A}(0) \\
\mathrm{S}(0.58) \\
\mathrm{B}(0.1,0.4) \\
\mathrm{B}(1.1,0.7) \\
1-150^{\circ} \text { (B-July) } \\
0.9-88.6^{\circ *}(29 \text { April })\end{array}$ & $\begin{array}{l}\mathrm{A}(0) \\
\mathrm{A}(0) \\
- \\
\mathrm{B}(0.3,0.5) \\
- \\
0.9-88.6^{\circ}(29 \text { April })\end{array}$ & $\begin{array}{l}A(0.01) \\
A(0) \\
B(0.3,0.5) \\
B(0.1,0.4) \\
0.9-160.9^{\circ} \\
0\end{array}$ \\
\hline Bursera fragrantissima & $\begin{array}{l}\text { Synchrony } \\
\text { Duration } \\
\text { Seasonality }\end{array}$ & $\begin{array}{l}\mathrm{A}(0.12) \\
\mathrm{A}(0.08) \\
\mathrm{B}(0.8,0.6) \\
\mathrm{B}(1.1,0.9) \\
0.9-111.9^{\circ} \\
0.2-62.6^{\circ}\end{array}$ & $\begin{array}{l}- \\
- \\
- \\
- \\
- \\
-\end{array}$ & $\begin{array}{l}\text { A }(0.47) \\
\text { A }(0.14) \\
\text { B }(0.9,0.4) \\
\text { B }(0.5,0.5) \\
1-120^{\circ} \\
1-180^{\circ *} \text { (31 July) }\end{array}$ & $\begin{array}{l}\mathrm{A}(0) \\
\mathrm{A}(0.08) \\
\mathrm{B}(0.2,0.4) \\
\mathrm{B}(0.5,0.5) \\
1-127.4^{\circ} \\
1-150^{\circ} \text { ( } 1 \text { July) }\end{array}$ & $\begin{array}{l}- \\
\mathrm{A}(0) \\
\overline{\mathrm{B}}(0.1,0.3) \\
\overline{-} \\
0.9-120^{\circ}\end{array}$ \\
\hline Caesalpinia coraria & $\begin{array}{l}\text { Synchrony } \\
\text { Duration } \\
\text { Seasonality }\end{array}$ & $\begin{array}{l}\text { A }(0.22) \\
\text { A }(0.11) \\
\text { B }(0.8,0.4) \\
\text { I }(2.9,2.6) \\
1-120.2^{\circ} \\
0.5-6.9^{\circ} \text { (6 February) }\end{array}$ & $\begin{array}{l}\mathrm{A}(0.04) \\
\mathrm{A}(0) \\
\mathrm{B}(1,1.2) \\
\mathrm{B}(1.7,1.9) \\
0.9-110.6^{\circ} \\
0.5-29.2^{\circ}(29 \\
\text { February) }\end{array}$ & $\begin{array}{l}\text { A }(0.08) \\
\text { A }(0.09) \\
\text { B }(0.6,0.7) \\
\text { I }(2.4,2.5) \\
1-125^{\circ} \\
0.7-6.2^{\circ *}(5 \text { February })\end{array}$ & $\begin{array}{l}\mathrm{A}(0.09) \\
\mathrm{A}(0.13) \\
\mathrm{B}(1.3,1.4) \\
\mathrm{B}(1.9,2.1) \\
0.9-114.4^{\circ} \\
0.7-4.4^{\circ *}(3 \\
\text { February) }\end{array}$ & $\begin{array}{l}\mathrm{A}(0.18) \\
\mathrm{A}(0.19) \\
\mathrm{B}(1.9,1.2) \\
\mathrm{I}(3,2.4) \\
0.8-125^{\circ} \\
0.6-15^{\circ}(14 \\
\text { February) }\end{array}$ \\
\hline Caesalpinia platyloba & $\begin{array}{l}\text { Synchrony } \\
\text { Duration } \\
\text { Seasonality }\end{array}$ & $\begin{array}{l}- \\
\mathrm{A}(0) \\
- \\
\mathrm{I}(2.1,2.4) \\
\overline{-} \\
0.6-64.4^{\circ}(4 \text { April })\end{array}$ & $\begin{array}{l}- \\
\mathrm{A}(0) \\
\overline{\mathrm{B}}(1.1,1.9) \\
\overline{0} \\
0.7-96.2^{\circ} \text { (7 May) }\end{array}$ & $\begin{array}{l}\mathrm{A}(0.12) \\
\mathrm{A}(0) \\
\mathrm{B}(0.6,0.5) \\
\mathrm{B}(1.8,3.4) \\
1-120^{\circ} \\
0.4-15^{\circ *}(14 \text { February })\end{array}$ & $\begin{array}{l}\bar{A}(0.16) \\
\overline{\mathrm{I}}(3.2,3.3) \\
\overline{0} \\
0.5-91^{\circ} \text { (1 May) }\end{array}$ & $\begin{array}{l}- \\
A(0.22) \\
- \\
E(5.2,3.9) \\
- \\
0.2-82.6^{\circ} \text { (23 April) }\end{array}$ \\
\hline Cordia elaeagnoides & $\begin{array}{l}\text { Synchrony } \\
\text { Duration } \\
\text { Seasonality }\end{array}$ & $\begin{array}{l}\mathrm{L}(0.46) \\
\mathrm{L}(0.42) \\
\mathrm{E}(8.4,0.8) \\
\mathrm{E}(7.1,0.9) \\
0.4-42.7^{\circ} \\
0.5-19.6^{\circ *}(19 \\
\text { February) }\end{array}$ & $\begin{array}{l}S(0.59) \\
S(0.6) \\
E(7.4,0.5) \\
I(4.2,0.8) \\
0.3-55.1^{\circ} \\
0.7-87.3^{\circ}(28 \text { April })\end{array}$ & $\begin{array}{l}S(0.64) \\
S(0.66) \\
E(7.4,0.7) \\
E(7.9,0.3) \\
0.3-2.1^{\circ} \\
0.4-16.1^{0^{*}}(15 \\
\text { February) }\end{array}$ & $\begin{array}{l}\mathrm{L}(0.4) \\
\mathrm{L}(0.34) \\
\mathrm{E}(5.2,1.5) \\
\mathrm{E}(6.6,1.3) \\
0.6-51.1^{\circ} \\
0.5-31^{\circ^{*}}(1 \text { March })\end{array}$ & $\begin{array}{l}\mathrm{L}(0.28) \\
\mathrm{A}(0.23) \\
\mathrm{E}(8.1,1.7) \\
\mathrm{I}(4.7,0.9) \\
0.3-49.3^{\circ} \\
0.6-17^{\circ}(16 \\
\text { February) }\end{array}$ \\
\hline Eysendhartia polystachya & $\begin{array}{l}\text { Synchrony } \\
\text { Duration } \\
\text { Seasonality }\end{array}$ & $\begin{array}{l}\mathrm{A}(0.02) \\
\overline{\mathrm{B}}(0.3,0.5) \\
\overline{1} \\
-30^{\circ} \\
-\end{array}$ & $\begin{array}{l}\mathrm{A}(0) \\
- \\
\mathrm{B}(0.1,0.3) \\
\overline{1-180^{\circ}} \text { (31 July) } \\
-\end{array}$ & $\begin{array}{l}- \\
- \\
- \\
- \\
-\end{array}$ & $\begin{array}{l}- \\
- \\
- \\
- \\
-\end{array}$ & $\begin{array}{l}- \\
- \\
- \\
- \\
-\end{array}$ \\
\hline Gliricidia sepium & $\begin{array}{l}\text { Synchrony } \\
\text { Duration } \\
\text { Seasonality }\end{array}$ & $\begin{array}{l}\mathrm{A}(0.18) \\
\mathrm{A}(0.22) \\
\mathrm{I}(3.1,1.7) \\
\mathrm{B}(1.8,0.4) \\
0.5-29.2^{\circ} \\
1-45^{\circ}(16 \text { March })\end{array}$ & $\begin{array}{l}\mathrm{L}(0.43) \\
\mathrm{A}(0) \\
\mathrm{I}(2.1,0.3) \\
\mathrm{B}(0.1(0.3) \\
1-47^{\circ} \\
1-30^{\circ}(29 \text { February })\end{array}$ & $\begin{array}{l}\mathrm{L}(0.38) \\
\mathrm{A}(0.05) \\
\mathrm{I}(2.6,1.2) \\
\mathrm{B}(1.1,1.2) \\
0.7-19.1^{\circ} \\
0.8-29.1^{\circ}(29 \\
\text { February) }\end{array}$ & $\begin{array}{l}\mathrm{L}(0.33) \\
\mathrm{A}(0.08) \\
\mathrm{I}(2.2,0.8) \\
\mathrm{B}(0.8,0.6) \\
0.9-31.8^{\circ} \\
1-26.2^{\circ}(26 \\
\text { February })\end{array}$ & $\begin{array}{l}\mathrm{A}(0.15) \\
\mathrm{A}(0.17) \\
\mathrm{B}(1.5,1.1) \\
\mathrm{B}(1.3,0.8) \\
0.8-26.1^{\circ} \\
1-41.5^{\circ}(12 \text { March })\end{array}$ \\
\hline Guazuma ulmifolia & $\begin{array}{l}\text { Synchrony } \\
\text { Duration } \\
\text { Seasonality }\end{array}$ & $\begin{array}{l}\mathrm{L}(0.37) \\
\mathrm{A}(0.18) \\
\mathrm{I}(2.8,0.6) \\
\mathrm{I}(4.3,3.3) \\
0.7-67.2^{\circ} \\
0.4-56.1^{\circ} \text { (27 March) }\end{array}$ & $\begin{array}{l}\mathrm{A}(0.01) \\
\overline{\mathrm{B}}(0.4,0.7) \\
\overline{1} \\
\overline{1-75^{\circ}} \\
-\end{array}$ & $\begin{array}{l}- \\
- \\
- \\
- \\
-\end{array}$ & $\begin{array}{l}A(0) \\
A(0) \\
B(0.1,0.3) \\
B(0.1,0.3) \\
1-150^{\circ *}(1 \text { July) } \\
1-0^{\circ}\end{array}$ & $\begin{array}{l}\mathrm{A}(0.03) \\
- \\
\mathrm{B}(0.7,1.1) \\
\overline{0} \\
0.9-73.3^{\circ} \\
-\end{array}$ \\
\hline $\begin{array}{l}\text { Handroanthus } \\
\text { impetiginosus }\end{array}$ & $\begin{array}{l}\text { Synchrony } \\
\text { Duration } \\
\text { Seasonality }\end{array}$ & $\begin{array}{l}\mathrm{A}(0.06) \\
\mathrm{A}(0.08) \\
\mathrm{B}(0.5,0.5) \\
\mathrm{B}(1.8,1.5) \\
1-0^{\circ} \\
0.7-62.2^{\circ}(2 \text { April })\end{array}$ & $\begin{array}{l}- \\
- \\
- \\
- \\
-\end{array}$ & $\begin{array}{l}- \\
- \\
- \\
- \\
-\end{array}$ & $\begin{array}{l}- \\
\mathrm{A}(0) \\
- \\
\mathrm{B}(0.3,0.5) \\
- \\
0.4-36.2^{\circ}\end{array}$ & $\begin{array}{l}- \\
- \\
- \\
- \\
-\end{array}$ \\
\hline
\end{tabular}


Table 2 (continued)

\begin{tabular}{|c|c|c|c|c|c|c|}
\hline Species & Attribute & 2010 & 2011 & 2012 & 2013 & 2014 \\
\hline Heteroflorum sclerocarpum & $\begin{array}{l}\text { Synchrony } \\
\text { Duration } \\
\text { Seasonality }\end{array}$ & $\begin{array}{l}\bar{A}(0.08) \\
\bar{I}(3.7,3.2) \\
\overline{0} \\
0.3-1.4^{\circ *} \text { (30 January) }\end{array}$ & $\begin{array}{l}\text { A }(0.02) \\
\text { A }(0.12) \\
\text { B }(0.3,0.5) \\
\text { I }(4,3.4) \\
1-90^{\circ} \\
0.4-76.8^{\circ^{*}}(17 \text { April })\end{array}$ & $\begin{array}{l}\mathrm{A}(0.02) \\
\mathrm{A}(0.0 .9) \\
\mathrm{B}(0.5,0.5) \\
\mathrm{I}(4.3,4.1) \\
0.9-90^{\circ} \\
0.4-9.2^{\circ *} \text { (8 February) }\end{array}$ & $\begin{array}{l}\mathrm{A}(0.02) \\
\mathrm{A}(0.05) \\
\mathrm{B}(0.3,0.5) \\
\mathrm{I}(3.2,3.6) \\
1-90^{\circ} \\
0.4-60^{\circ}(1 \text { April })\end{array}$ & $\begin{array}{l}- \\
\mathrm{A}(0) \\
- \\
\mathrm{B}(1.6,2.1) \\
- \\
0.7-45^{\circ} \text { (16 March) }\end{array}$ \\
\hline Licania arborea & $\begin{array}{l}\text { Synchrony } \\
\text { Duration } \\
\text { Seasonality }\end{array}$ & $\begin{array}{l}- \\
- \\
- \\
- \\
-\end{array}$ & $\begin{array}{l}\mathrm{A}(0) \\
- \\
\mathrm{B}(0.1,0.3) \\
- \\
1-90^{\circ *}(30 \text { April }) \\
-\end{array}$ & $\begin{array}{l}\mathrm{A}(0) \\
- \\
\mathrm{B}(0.1,0.3) \\
- \\
1-30^{\circ *}(29 \text { February }) \\
-\end{array}$ & $\begin{array}{l}\mathrm{A}(0.05) \\
\mathrm{A}(0.05) \\
\mathrm{B}(0.4,0.5) \\
\mathrm{B}(0.4,0.8) \\
1-30^{\circ} \\
1-80.1^{\circ}\end{array}$ & $\begin{array}{l}\mathrm{A}(0.19) \\
\mathrm{A}(0.19) \\
\mathrm{I}(2.3,1.2) \\
\mathrm{B}(0.4,0.5) \\
0.9-39^{\circ} \\
1-39.9^{\circ}\end{array}$ \\
\hline Plumeria rubra & $\begin{array}{l}\text { Synchrony } \\
\text { Duration } \\
\text { Seasonality }\end{array}$ & $\begin{array}{l}\text { A }(0.24) \\
\text { A }(0.15) \\
\text { I }(3.2,0.8) \\
\text { I }(4.2,2) \\
0.9-136^{\circ} \\
0.2-0.3^{\circ *} \text { (30 January) }\end{array}$ & $\begin{array}{l}\mathrm{A}(0.22) \\
\mathrm{A}(0.07) \\
\mathrm{I}(2.7,1.2) \\
\mathrm{B}(1.6,1.4) \\
0.9-106.3^{\circ} \\
0.5-38.3^{0^{*}}(9 \text { March })\end{array}$ & $\begin{array}{l}\mathrm{A}(0.25) \\
\mathrm{A}(0.23) \\
\mathrm{I}(4,1.1) \\
\mathrm{B}(1.7,0.8) \\
0.7-113.4^{\circ} \\
0.8-21.9^{\circ}(21 \\
\text { February) }\end{array}$ & $\begin{array}{l}\mathrm{A}(0.23) \\
\mathrm{A}(0.24) \\
\mathrm{I}(3.6,1.3) \\
\mathrm{I}(4.9,1) \\
0.8-122.1^{\circ} \\
0.6-33.5^{\circ} \text { (4 March) }\end{array}$ & $\begin{array}{l}\mathrm{A}(0.32) \\
\mathrm{A}(0.14) \\
\mathrm{I}(3.1,0.9) \\
\mathrm{B}(1.3,0.9) \\
0.8-122.5^{\circ} \\
1-15^{\circ}\end{array}$ \\
\hline Swietenia humilis & $\begin{array}{l}\text { Synchrony } \\
\text { Duration } \\
\text { Seasonality }\end{array}$ & $\begin{array}{l}\mathrm{A}(0.09) \\
\mathrm{A}(0) \\
\mathrm{B}(0.8,0.9) \\
\mathrm{B}(0.8,0.9) \\
1-39.9^{\circ} \\
1-15^{\circ}(14 \text { February })\end{array}$ & $\begin{array}{l}\mathrm{A}(0.01) \\
- \\
\mathrm{B}(0.3,0.5) \\
- \\
1-30^{\circ *}(29 \text { February }) \\
-\end{array}$ & $\begin{array}{l}\mathrm{A}(0.02) \\
\mathrm{A}(0.1) \\
\mathrm{B}(0.3,0.5) \\
\mathrm{B}(0.9,1.2) \\
1-30^{\circ *}(29 \text { February }) \\
0.9-38.8^{\circ *}(9 \text { March })\end{array}$ & $\begin{array}{l}\mathrm{A}(0) \\
\mathrm{A}(0) \\
\mathrm{B}(0.1,0.4) \\
- \\
1-30^{\circ}(29 \text { February }) \\
0.1-6.2^{\circ}\end{array}$ & $\begin{array}{l}- \\
\mathrm{A}(0) \\
- \\
- \\
- \\
0.9-88.6^{\circ}\end{array}$ \\
\hline
\end{tabular}

$R$ ( $R$ Core Team, 2014). We calculated the coefficient of discrimination (D), a measure of the model's ability to discriminate failures and successes in a binary response variable (Tjur, 2009). Because of collinearity between temperature and photoperiod $(r=0.64)$, temperature data were excluded from further analyses.

\section{Results}

\subsection{Reproductive phenological patterns}

\subsubsection{Intensity and frequency}

Overall, reproductive phenophases occurred with mean low intensity $(<25 \%)$ in our study species (Fig. 2). The large variation between years and species makes it difficult to identify the moment of the year with flowering and fruiting peaks. Nevertheless, the reproductive period was strongly concentrated in the dry season (October-May). All species showed a considerable time lag between the presence of flowers and fruits, i.e., flowers were commonly recorded one to three months prior to the production of mature fruits, except in Cordia elaeagnoides and Swietenia humilis, for which there was a temporal overlap in the occurrence of the two phenophases (Fig. 2). In the case of Caesalpinia platyloba, in four of five years, fruits were observed without any previous record of flowering (missing observation probably related to very short less than two weeks - flowering event), and the opposite happened with Eysendhartia polystachya, for which only two incipient flowering events were observed, but not a single fruiting event, during the entire study period.

The reproductive cycles of nine study species were annual, while the remaining five were classified as supra-annual (E. polystachya, Guazuma ulmifolia, Handroanthus impetiginosus, Licania arborea and S. humilis), as for these species flowers and fruits were not recorded in all consecutive years of the study. All nine annual species exhibited the same phenological pattern year after year, albeit with small variations in their timing and intensity (Fig. 2).

Regardless of reproductive frequency in our study species, three main phenological strategies were identified based on their flowering and fruiting time and intensity: (1) flowering and fruiting exclusively during the rainy season (Bursera fragrantissima), (2) flowering in the rainy season and fruiting in the dry season (Amphipterygium adstringens, Brosimum alicastrum, C. elaeagnoides and C. platyloba), and (3) flowering and fruiting exclusively occurring during the dry season (Caesalpinia coriaria, Gliricidia sepium, Heteroflorum sclerocarpum, L. arborea, Plumeria rubra and S. humilis). Three species could not be classified in any of these groups due to the erratic and incipient patterns in their reproductive phenology (E. polystachya, G. ulmifolia and H. impetiginosus).

\subsubsection{Synchrony}

Overall, flowering and fruiting of the study species were not synchronic (Table 2), with mean synchrony indices of 0.1 and 0.06 , respectively. The analysis of the patterns between years and species revealed important differences. The only species that flowered and fruited synchronously during the five-year period was $C$. elaeagnoides (mean synchrony index of 0.5 for both phenophases). Other species showed some degree of synchrony in one phenophase but considerable asynchrony in the other. For example, flowering of $G$. sepium was moderately synchronic in three years, whereas its fruiting was always asynchronic, in contrast with B. alicastrum, in which flowering was asynchronic in all the years of study while fruiting was much more synchronic.

\subsubsection{Duration}

Reproductive structures generally remained on the trees less than two months (Table 2); generally, flowers had a shorter duration than fruits (means \pm SD: $1.2 \pm 2$ and $2.1 \pm 1.4$ months, respectively). When considering inter-annual and interspecific variation, $C$. elaeagnoides was the only species whose flowers and fruits were not only more synchronous in their appearance, but also fruits remained on the trees for over five months. In other species, the durations of the two phenophases were different. For example, flowers last longer than fruits in G. sepium and P. rubra, whilst in A. adstringens, C. coriaria, and H. sclerocarpum, the opposite was true, with a short flowering and an intermediate fruiting. All species classified as supra-annual retained their flowers and fruits for short periods (mean \pm SD: $0.5 \pm 0.5$ months) 
Table 3

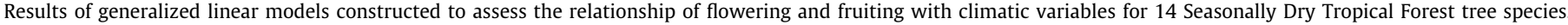

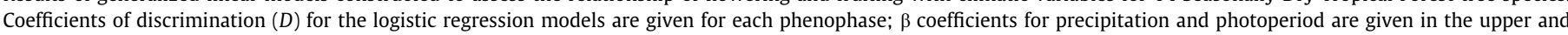
lower row of each model, respectively.

\begin{tabular}{|c|c|c|c|c|}
\hline Species & Phenophase & $D$ & $\beta$ & $Z$ \\
\hline Amphipterygium adstringens & $\begin{array}{l}\text { Flowering } \\
\text { Fruiting }\end{array}$ & $\begin{array}{l}0.13 \\
0.06\end{array}$ & $\begin{array}{l}0.002 \\
6.281 \\
0.001 \\
-0.835\end{array}$ & $\begin{array}{l}2.57^{* *} \\
11.25^{* * *} \\
5.80^{* * *} \\
-16.70^{* * *}\end{array}$ \\
\hline Brosimum alicastrum & $\begin{array}{l}\text { Flowering } \\
\text { Fruiting }\end{array}$ & $\begin{array}{l}0.03 \\
0.04\end{array}$ & $\begin{array}{l}0.006 \\
0.364 \\
-0.003 \\
-1.406\end{array}$ & $\begin{array}{l}10.21^{* * * *} \\
2.26^{\text {n.s. }} \\
-2.40^{* * *} \\
-9.70^{* * *}\end{array}$ \\
\hline Bursera fragrantissima & $\begin{array}{l}\text { Flowering } \\
\text { Fruiting }\end{array}$ & $\begin{array}{l}0.07 \\
0.01\end{array}$ & $\begin{array}{l}-0.004 \\
2.048 \\
0.003 \\
1.070\end{array}$ & $\begin{array}{l}-5.24^{* * *} \\
13.08^{* * *} \\
4.02^{* * *} \\
5.80^{* * *}\end{array}$ \\
\hline Caesalpinia coriaria & $\begin{array}{l}\text { Flowering } \\
\text { Fruiting }\end{array}$ & $\begin{array}{l}0.13 \\
0.06\end{array}$ & $\begin{array}{l}0.003 \\
-0.922 \\
-0.628 \\
-0.010\end{array}$ & $\begin{array}{l}7.33^{* * * *} \\
-12.13^{* * * *} \\
-8.70^{* * * *} \\
-7.40^{* * * *}\end{array}$ \\
\hline Caesalpinia platyloba & $\begin{array}{l}\text { Flowering } \\
\text { Fruiting }\end{array}$ & $\begin{array}{l}0.06 \\
0.04\end{array}$ & $\begin{array}{l}-0.011 \\
2.859 \\
-0.003 \\
0.977\end{array}$ & $\begin{array}{l}-8.83^{* * *} \\
11.30^{* * * *} \\
-8.50^{* * *} \\
14.30^{* * * *}\end{array}$ \\
\hline Cordia elaeagnoides & $\begin{array}{l}\text { Flowering } \\
\text { Fruiting }\end{array}$ & $\begin{array}{l}0.06 \\
0.05\end{array}$ & $\begin{array}{l}0.001 \\
-1.246 \\
0.006 \\
-1.365\end{array}$ & $\begin{array}{l}26.30^{* * *} \\
-4.67^{* * *} \\
12.27^{* * *} \\
-23.95^{* * *}\end{array}$ \\
\hline Eysendharthia polystachya & $\begin{array}{l}\text { Flowering } \\
\text { Fruiting }\end{array}$ & $\begin{array}{l}0.006 \\
-\end{array}$ & $\begin{array}{l}0.001 \\
0.857 \\
- \\
-\end{array}$ & $\begin{array}{l}0.98^{\text {n.s. }} \\
4.50^{* * *} \\
- \\
-\end{array}$ \\
\hline Gliricidia sepium & $\begin{array}{l}\text { Flowering } \\
\text { Fruiting }\end{array}$ & $\begin{array}{l}0.006 \\
0.03\end{array}$ & $\begin{array}{l}-0.019 \\
-1.594 \\
-0.041 \\
-0.825\end{array}$ & $\begin{array}{l}-6.70^{* * *} \\
-6.40^{* * *} \\
-11.35^{* * * *} \\
-18.76^{* * *}\end{array}$ \\
\hline Guazuma ulmifolia & $\begin{array}{l}\text { Flowering } \\
\text { Fruiting }\end{array}$ & $\begin{array}{l}0.05 \\
0.004\end{array}$ & $\begin{array}{l}-0.009 \\
1.520 \\
-0.004 \\
0.541\end{array}$ & $\begin{array}{l}-11.69^{* * * *} \\
15.50^{* * * *} \\
-3.60^{* * * *} \\
3.70^{* * *}\end{array}$ \\
\hline Handroanthus impetiginosus & $\begin{array}{l}\text { Flowering } \\
\text { Fruiting }\end{array}$ & $\begin{array}{l}0.006 \\
0.02\end{array}$ & $\begin{array}{l}0.005 \\
-0.303 \\
0.028 \\
-1.375\end{array}$ & $\begin{array}{l}7.51^{* * *} \\
-1.84^{\text {n.s. }} \\
-5.50^{* * *} \\
6.30^{* * *}\end{array}$ \\
\hline Heteroflorum sclerocarpum & $\begin{array}{l}\text { Flowering } \\
\text { Fruiting }\end{array}$ & $\begin{array}{l}0.17 \\
0.02\end{array}$ & $\begin{array}{l}0.005 \\
-0.303 \\
-0.028 \\
1.375\end{array}$ & $\begin{array}{l}-13.69^{* * * *} \\
14.11^{* * * *} \\
-3.02^{* *} \\
-7.20^{* * * *}\end{array}$ \\
\hline Licania arborea & $\begin{array}{l}\text { Flowering } \\
\text { Fruiting }\end{array}$ & $\begin{array}{l}0.06 \\
0.007\end{array}$ & $\begin{array}{l}-0.005 \\
1.984 \\
-0.030 \\
1.800\end{array}$ & $\begin{array}{l}-2.57^{* * *} \\
-9.64^{* * *} \\
-3.40^{* * *} \\
-4.30^{* * * *}\end{array}$ \\
\hline Plumeria rubra & $\begin{array}{l}\text { Flowering } \\
\text { Fruiting }\end{array}$ & $\begin{array}{l}0.05 \\
0.03\end{array}$ & $\begin{array}{l}-0.009 \\
3.404 \\
-0.006 \\
-0.644\end{array}$ & $\begin{array}{l}-17.11^{* * * *} \\
28.59^{* * * *} \\
-6.20^{* * *} \\
-6.90^{* * *}\end{array}$ \\
\hline Swietenia humilis & $\begin{array}{l}\text { Flowering } \\
\text { Fruiting }\end{array}$ & $\begin{array}{l}0.05 \\
0.008\end{array}$ & $\begin{array}{l}-0.130 \\
0.745 \\
-0.011 \\
0.403\end{array}$ & $\begin{array}{l}-5.21^{* * *} \\
6.16^{* * *} \\
-5.05^{* * *} \\
2.40^{* *}\end{array}$ \\
\hline
\end{tabular}

n.s. - not significant.

${ }^{* *} P<0.01$.

${ }^{* * *} P<0.001$.

\subsubsection{Seasonality}

The reproductive events of the study species occurred mostly in the dry season; however, the statistical analysis of seasonality did not show significant results for the flowering of any species, even though some had a significant mean $\alpha$ in one year (Table 2). For example, A. adstringens, B. alicastrum, G. ulmifolia and E. polystachya flowered in the rainy season, between June and August, while other species like $L$. arborea and $S$. humilis had flowers in the dry season (March-May).

Conversely, fruiting seasonality was significant in the five study years for C. platyloba, C. coriaria, $C$. elaeagnoides and $H$. sclerocarpum. For the former species, mean angle occurred between April 
and May (one year in February), and for the latter three species it did so between February and April. Fruits of G. sepium and P. rubra were also observed in this latter period, but seasonality was significant in four out of five years (Table 2). As expected, those species that retained their reproductive structures for a long time did not present a significant seasonality, as exemplified by C. elaeagnoides.

\subsection{Relation between reproductive phenology and climatic variables}

The signs of the relationships between phenophases and environmental factors showed complex patterns across species (Table 3). In some species, flowering and fruiting showed a similar relationship (i.e. positive or negative) with precipitation, and the same was true for photoperiod. This situation is exemplified by G. ulmifolia and S. humilis, whose two phenophases were negatively related with precipitation, but positively with photoperiod (dry season, March to April), and by G. sepium and L. arborea, for which flowering and fruiting were negatively related with the two environmental factors. In other cases, one phenophase displayed contrasting relationships with precipitation and photoperiod, while the other showed similar relationships. For example, the flowering of $A$. adstringens was positively related both with rain and photoperiod (rainy season, May to July), whilst fruiting was positively related with the former factor but negatively with the latter. Also, the fruiting of $B$. fragrantissima was positively related with both environmental variables, whereas its flowering had contrasting relationships with them.

Finally, some species stood out from these patterns, as their flowering was significantly related with one environmental variable but not with the other. For example, in $B$. alicastrum and $H$. impetiginosus flowering was positively related with precipitation only, whereas in E. polystachia, a species for which no fruiting was observed throughout the five year monitoring period, flowering was unrelated with rainfall but positively related with photoperiod (Table 3).

\section{Discussion}

\subsection{Reproductive phenological strategies and climatic clues}

The flowering and fruiting of all species concentrated strongly either in the early (October-November) or the late (FebruaryMarch) dry season. Overall, both phenophases had a low intensity $(<25 \%)$, a short duration ( $<2$ months), were asynchronic and had an annual frequency. This general pattern largely coincides with reports for other SDTF species (e.g., Bullock and Solís-Magallanes, 1990; Borchert et al., 2004; Valdez-Hernández et al., 2010). Yet, the specific responses or phenological strategies to climatic seasonality during the year diverged, with the predominance of dry season phenological strategy, which includes the species reproducing exclusively during the dry season (Table 4; October-March). That strategy indicates a clear adaptation of those STDF trees to the strong precipitation seasonality, and it has been observed for other tropical dry forests (Singh and Kushwaha, 2005). Species presenting this strategy may remain dormant until leaf shedding occurs and the trunk rehydrates, allowing flower anthesis and fruit maturation (Borchert, 2000). Species in this study conforming to the above strategy (e.g., A. adstringens, B. alicastrum, C. elaeagnoides and $C$. platyloba) show a fruiting peak in the early dry season (October-November), but their fruits stayed on the trees for over five months, which resulted in the lack of statistically significant seasonality. The second most common phenological strategy grouped five species flowering in the rainy season (June-August) and fruiting in the dry season (September-November). Only one species presented the third phenological strategy, flowering and bearing fruits exclusively during the wet season (JuneSeptember), indicating a sensitivity to the dry season environmental constraints. Species with this latter strategy, in our study represented by $B$. fragrantissima, respond rapidly to increasing rainfall, usually have a single, short ( $<2$ months) flowering event each year; flower buds may mature quickly, and fruits are produced at the end of the same season, when dispersers abound (JulySeptember; van Schaik et al., 1993). Therefore, phenological patterns of these STDF tree species are strongly related with precipitation seasonality, and thus deviations in the starting dates and intensity of the reproductive phenophases may be observed in response to large inter-annual variation in precipitation.

For those species that began to flower during the dry season, photoperiod may be the trigger of the reproductive activity, as they can either uptake soil water through a deep root system, or use water previously stored in low wood density trunks (Borchert and Rivera, 2001; Borchert et al., 2004; Rivera et al., 2002). Species displaying this dry season phenological strategy tend to flower briefly ( $<2$ months), two to three months after the end of the rains when photoperiod becomes shorter (Fall, November-December) like $G$. sepium and $C$. coriaria, or several weeks before the onset of the wet period, when photoperiod is longer (Spring, MarchApril), like H. sclerocarpum, L. arborea, P. rubra and S. humilis. When

Table 4

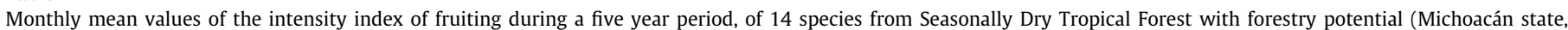
Mexico). Bold values indicate the period in which it is recommended to collect their seeds according to their phenological patterns.

\begin{tabular}{|c|c|c|c|c|c|c|c|c|c|c|c|c|}
\hline Phenological pattern/Species & January & February & March & April & May & June & July & August & September & October & November & December \\
\hline \multicolumn{13}{|c|}{ Flowering and fruiting in the rainy season } \\
\hline Bursera fragrantissima & 0 & 0 & 2.6 & 0 & 1 & 0 & 7.2 & 7.8 & 2.8 & 0 & 0 & 0 \\
\hline \multicolumn{13}{|c|}{ Flowering in rains, fruiting in drought } \\
\hline Amphipterygium adstringens & 38 & 32.5 & 24.4 & 14.4 & 6 & 0.8 & 10 & 34 & 47.6 & 48.4 & 38 & 19.2 \\
\hline Brosimum alicastrum & 0.8 & 0.7 & 0.5 & 3.2 & 1.6 & 0.3 & 0 & 0.5 & 0.8 & 3.2 & 31.2 & 5.9 \\
\hline Cordia elaeagnoides & 54.8 & 53.5 & 31.6 & 22 & 10.8 & 2 & 0 & 0 & 10 & 64 & 62.8 & 46.4 \\
\hline Caesalpinia platyloba & 19.2 & 14 & 10 & 8.4 & 3.6 & 0.8 & 6.8 & 16.4 & 16.8 & 18.4 & 19.2 & 20.8 \\
\hline \multicolumn{13}{|c|}{ Flowering and fruiting in the dry season } \\
\hline Gliricidia sepium & 0 & 4.5 & 14.4 & 9.2 & 0.4 & 0 & 0 & 0 & 0 & 0 & 0 & 0.8 \\
\hline Caesalpinia coriaria & 26.8 & 30.5 & 15.6 & 6.8 & 4 & 0.4 & 1.6 & 0.4 & 0.4 & 0.8 & 2.8 & 10 \\
\hline Heteroflorum sclerocarpum & 13.6 & 15.5 & 16.4 & 12.8 & 7.6 & 1.6 & 0 & 3.6 & 12.4 & 14 & 13.2 & 12 \\
\hline Licania arborea & 0 & 0 & 1.1 & 1.7 & 1.1 & 0 & 0 & 0 & 0 & 0 & 0 & 0 \\
\hline Plumeria rubra & 13.2 & 16.5 & 14.4 & 6 & 1.6 & 0 & 0.4 & 0 & 3.6 & 7.6 & 6.4 & 8 \\
\hline Swietenia humilis & 0 & 5 & 7 & 5 & 0 & 0 & 0 & 0 & 0 & 0 & 0 & 0 \\
\hline \multicolumn{13}{|l|}{ Uncertain pattern } \\
\hline Eysendharthia polystachya & 0 & 0 & 0 & 0 & 0 & 0 & 0 & 0 & 0 & 0 & 0 & 0 \\
\hline Guazuma ulmifolia & 1.2 & 3.5 & 4 & 7.6 & 2 & 0.4 & 2 & 0.8 & 0.8 & 0.8 & 0.4 & 0.4 \\
\hline Handroanthus impetiginosus & 0.4 & 0 & 2 & 4 & 3.6 & 0 & 0.4 & 0 & 0 & 0.4 & 0 & 1.2 \\
\hline
\end{tabular}


flowering occurs in the middle of the dry season, fruit availability will peak shortly after (January-March). Conversely, if species bloom in the late dry season, they will initiate fruit production two-three months later, and fruits will remain in the trees for a variable time (3-9 months), which ensures that their seeds will be ready for dispersal just before the next rainy season (AprilMay). As photoperiod fluctuations are constant from year to year, the reproductive phenological patterns of these species should display small inter-annual variations.

Admittedly, the ability of the logistic models to efficiently discriminate failures and successes (i.e., presence or absence of reproductive structures) in our study species was modest. Although this result could imply that the environmental factors play a relatively minor role in the timing of phenological events, this is more likely due to the reduction of the observed phenological variability to a binary variable for this analysis. Yet, the importance of this result should not be disregarded, considering that the large majority of phenological studies comprising many species over long periods of time produce this kind of information.

\subsection{Reproductive phenology asynchrony}

Due to the large inter-annual variation in precipitation in the seasonally dry tropics, species with reproductive cycle responding to increasing water availability may have a more asynchronic phenology, compared with the constancy of species whose reproduction is triggered by photoperiod (Borchert et al., 2005; Singh and Kushwaha, 2006). Interestingly, our findings disagree with these generalizations: regardless of their phenological pattern, all species but one ( $C$. elaeagnoides) were highly asynchronic in their flowering and fruiting. This suggests that despite strong environmental clues that may trigger their reproduction, morphological and physiological intra-specific variability may produce differential responses among individuals to micro environmental conditions (Holbrook et al., 1995; Singh and Kushwaha, 2005; van Schaik et al., 1993). The synchrony index used in this study takes into account both the degree of overlap between different phenophases and their intensity; thus, a high synchrony requires the phenophase peak in a given individual to coincide with the peaks of all its conspecifics (Freitas and Bolmgren, 2008). As the reproductive intensity recorded by us was generally low, this also resulted in low synchrony.

\subsection{Seed collection strategies and phenological patterns}

In SDTFs, plant propagation in rustic nurseries is confined to the rainy season. The relevance of a good seed collection schedule for the proper functioning of the nurseries resides thereby, as it ensures a timely seed supply (Blakesley et al., 2002; Böhringer et al., 2003). Seed collection planning requires precise knowledge of phenological patterns, as this allows deciding the time of the year when it is more likely to find mature fruits for the focal species, and helps understand the close relationship between reproductive cycles of plants and the typically changing climatic conditions in dry forest regions (Buisson et al., 2016; Morellato et al., 2016). Regrettably, phenology-informed seed collecting agendas are often constructed based on short-term studies in which different phenological attributes are not evaluated quantitatively (Buisson et al., 2016). A major drawback of this approach is that it results in inflexible collecting schemes that prevent adapting to the intrinsic inter-annual phenological variability. Our study exemplifies this situation: in the first monitoring year a highintensity, three day-long rainfall event took place in the middle of February, a typically dry month, which strongly modified the usual reproductive pattern of species that respond rapidly to water inputs. Consequently, in that year flowering began early and it was followed by flower bud abortion due to insufficient water in subsequent months. Our approach provided essential elements to translate the observed phenological patterns into adaptive management plans.

For species that concentrate their reproductive cycle in the rainy season one may be confident that fruit availability will be high in July and August. However, because flowering and fruiting in these species tend to be very short (usually $<1$ month), frequent monitoring of their trees (at least fortnightly) will increase the probability to find fruits for seed collection. The trade-offs related to frequency of observation and sample sizes have been discussed by Morellato et al. (2010) and support our findings. In our study, $B$. fragantissima was the only species in this situation: in 2011 we failed to record flowers and fruits, probably due to an insufficient temporal resolution in the monitoring schedule rather than because of a supra-annual pattern in its reproductive cycle. Species that flower in the rainy season represent a contrasting situation; many of them bore immature fruits already in September, and they became mature one or two months later, in the early dry season. For these species it would be advisable to collect their seeds at this time, regardless of how long their fruits stay on the trees, given the considerable reductions in seed viability reported after this period (Fenner and Thompson, 2004).

The flowering and fruiting of any individual plant are not independent events: the onset of the flowering largely determines the timing of fruiting (Primack, 1987; Singh and Kushwaha, 2005). For those species that display any of the first two phenological strategies described here, recording flower initiation in any given year is very relevant, as one may forecast one or two months ahead of time when seeds will be available. Likewise, for species flowering and fruiting exclusively in the dry season, either because their reproductive cycle responds to a decreasing or increasing photoperiod, we recommend to collect their seeds in February and March (Table 4).

When planning seed collection one must consider other relevant issues in addition to the time schedule of this activity. For example, it is also necessary to know the sexual system of target species; for dioecious species one should select individuals to be monitored carefully to prevent the inclusion of male trees only. Information on the spatial distribution and abundance patterns of focal species is also important: collecting seeds of rare species that are widely spread spatially will have very different logistic requirements compared to abundant species or those having clumped spatial patterns (Buisson et al., 2016; Morellato et al., 2016).

To close, we emphasize that the success of a long-term phenological study, like the one presented here, largely depends on the coordinated participation of the various social actors involved in seed management of native species, including local communities, government agencies, private initiatives and the academia. In our experience, the implementation of the community-based monitoring ( $\mathrm{CBM})$ program allowed local stakeholders to become familiar with the underlying causes of the large variability in the observed phenological patterns, and to acquire basic abilities that will allow them to conduct an adaptive management of seeds of native species in this highly dynamic system. The CBM represents a prime example of the citizen science approach (Burgos et al., 2013; Dickinson et al., 2010; Kobori et al., 2016), which will allow the community to use wisely and in a more sustainable way the natural resources they have traditionally extracted from the STDF (Balvanera et al., 2011). Ideally, our study will serve to stimulate citizen science in the tropics, as this approach would offer important avenues for future studies in phenology and conservation science, as well as in education and management programs (Buisson et al., 2016; Mayer, 2010; Morellato et al., 2016; Scheifinger and Templ, 2016). 


\section{Conclusions}

Long-term phenological studies that include qualitative descriptions of population-level phenological attributes provide valuable information needed to understand both the inter-annual and interspecific variation implicit in the phenological patterns of tree species. Understanding this variation is critical for the design of flexible seed collection strategies that can adapt to the intrinsic variations of plants' life cycles, and thus to ensure a timely and permanent provision of seed to multiple programs aimed at the propagation of native species of forestry interest.

\section{Acknowledgements}

The senior author thanks the Posgrado en Ciencias Biologicas of Universidad Nacional Autónoma de México for its support during her PhD studies and CONACYT for a graduate scholarship. This article is a requirement for obtaining the $\mathrm{PhD}$ degree. This research was funded by PAPIIT-UNAM, grant IN207512. Jorge Cortés Flores provided assistance in statistical analysis. This paper benefited from the constructive and useful comments from one anonymous reviewer.

\section{Appendix A}

\section{See Table A1.}

Table A1

Total number of individuals of 14 tree species recorded within the study area in Churumuco, Michoacán state, Mexico. Sites (m): Low (230-478), Medium (479-726) and High (727-974).

\begin{tabular}{lllll}
\hline Species & Low & Medium & High & Total \\
\hline Amphipterygium adstringens & 3 & 38 & 45 & 86 \\
Brosimum alicastrum & - & 16 & 12 & 28 \\
Bursera fragrantissima & - & - & 50 & 50 \\
Caesalpinia coriaria & 10 & 1 & - & 11 \\
Caesalpinia platyloba & 8 & 32 & - & 40 \\
Cordia elaeagnoides & 42 & 49 & 20 & 111 \\
Eysenhardtia polystachya & - & - & 28 & 28 \\
Gliricidia sepium & 40 & 19 & 2 & 61 \\
Guazuma ulmifolia & 2 & 5 & 56 & 63 \\
Handroanthus impetiginosus & 19 & 32 & 4 & 55 \\
Heteroflorum sclerocarpum & 18 & 31 & - & 49 \\
Licania arborea & 2 & 3 & - & 5 \\
Plumeria rubra & - & 37 & - & 37 \\
Swietenia humilis & - & 1 & 10 & 11 \\
Total & 144 & 264 & 227 & 635 \\
\hline
\end{tabular}

\section{References}

Balvanera, P., Castillo, A., Martínez-Harms, M.J., 2011. Ecosystem services in seasonally dry tropical forests. In: Dirzo, R., Young, H.S., Mooney, H.A., Ceballos, G. (Eds.), Seasonally Dry Tropical Forests. Ecology and Conservation. Island Press, Washington, DC, pp. 259-277.

Blakesley, D., Elliot, S., Kuarak, C., Navakitbumrung, P., Zangkum, S., Anunsarnsunthorn, V., 2002. Propagating framework tree species to restore seasonally dry tropical forest: implication of seasonal seed dispersal and dormancy. For. Ecol. Manage. 164, 31-38.

Böhringer, A., Ayuk, E.T., Katanga, R., Ruvuga, S., 2003. Farmer nurseries as a catalyst for developing sustainable land use systems in southern Africa. Part A: Nursery productivity and organization. Agric. Syst. 77, 187-201.

Bonfil, C., Trejo, I., 2010. Plant propagation and the ecological restoration of Mexican tropical deciduous forest. Ecol. Rest. 28, 369-376.

Borchert, R., 1998. Responses of tropical trees to rainfall seasonality and its longterm changes. Clim. Change 39, 381-393.

Borchert, R., 2000. Organismic and environmental controls of bud growth in tropical trees. In: Viemont, J.D., Crabbé, J. (Eds.), Dormancy in Plants: From Whole Plant Behavior to Cellular Control. CAB International, Wallingford, pp. 87-107.

Borchert, R., Rivera, G., 2001. Photoperiodic control of seasonal development and dormancy in tropical stem succulent trees. Tree Physiol. 21, 213-221.
Borchert, R., Rivera, G., Hagnauer, W., 2002. Modification of vegetative phenology in a tropical semi-deciduous forest by abnormal drought and rain. Biotropica 34 27-39.

Borchert, R., Meyer, S.A., Felger, R.S., Porter-Bolland, L., 2004. Environmental control of flowering periodicity in Costa Rican and Mexican tropical dry forests. Global Ecol. Biogeogr. 13, 409-425.

Borchert, R., Renner, S.S., Calle, Z., Navarrete, D., Tye, A., Gautier, L., Spichiger, R., von Hildebrand, P., 2005. Photoperiodic induction of synchronous flowering near equator. Nature 433, 627-629.

Botha, J., Witkowski, E.T.F., Cock, J., 2005. A review of nurseries as conservation or social forestry outreach tools. Int. J. Biodivers. Sci. Manage. 1, 33-51.

Botha, J., Witkowski, E.T.F., Cock, J., 2006. The South African experience of conservation and social forestry outreach nurseries. Environ. Manage. 38, 733-749.

Buisson, E., Alvarado, S.T., Stradic, S.L., Morellato, P., 2016. Plant phenological research enhances ecological restoration. Restoration Ecol. http://dx.doi.org/ $10.1111 /$ rec. 12471.

Bullock, S.H., Solís-Magallanes, J.A., 1990. Phenology of canopy trees of a tropical deciduous forest in Mexico. Biotropica 22, 22-35.

Burgos, A., Páez, R., Carmona, E., Rivas, H., 2013. A system approach to modeling Community-Based Environmental Monitoring: a case of participatory water quality monitoring in rural Mexico. Environ. Monit. Assess. 185, 10297-10316.

Cortés-Flores, J., Hernández-Esquivel, K.B., González-Rodríguez, A., IbarraManríquez, G., 2017. Flowering phenology, growth forms and pollination syndromes in a tropical dry forest species: influence of phylogeny and abiotic factors. Am. J. Bot. 104, 39-49.

Dickinson, J.L., Zuckerberg, B., Bonter, D.N., 2010. Citizen science and ecological research tool: challenges and benefits. Ann. Rev. Ecol. Evol. Syst. 41, 149-172.

Fenner, M., Thompson, K., 2004. The Ecology of Seeds. Cambridge University Press, Cambridge.

Fournier, L.A., 1974. Un método cuantitativo para la medición de características fenológicas en árboles. Turrialba 24, 422-423.

Frankie, G.W., Baker, H.G., Opler, P.A., 1974. Comparative phenological studies of trees in tropical wet and dry forests in the lowlands of Costa Rica. J. Ecol. 62, 881-919.

Freitas, L., Bolmgren, K., 2008. Synchrony is more than overlap: measuring phenological synchronization considering time length and intensity. Rev. Bras. Bot. 31, 721-724.

Holbrook, N.M., Whitbeck, J.L., Mooney, H.A., 1995. Drought responses of Neotropical dry forest trees. In: Bullock, S.H., Mooney, H.A., Medina, E. (Eds.) Seasonally Dry Tropical Forests. Cambridge University Press, Cambridge, pp. 243-276.

Justiniano, M.J., Fredericksen, T.S., 2000. Phenology of tree species in Bolivian dry forest. Biotropica 32, 276-281.

Kobori, H., Dickinson, J.L., Washitani, I., Sakurai, R., Amano, T., Komatsu, N., Kitamura, W., Takagawa, S., Koyama, K., Ogawara, T., Miller-Rushing, A.J., 2016. Citizen science: a new approach to advance ecology, education and conservation. Ecol. Res. 31, 1-19.

Kuaraksa, C., Elliot, S., Hossaert-Mckey, M., 2012. The phenology of dioecious Ficus spp. tree species and its importance for forest restoration projects. For. Ecol. Manage. 265, 82-93.

Mayer, A., 2010. Phenology and citizen science. BioScience 60, 172-175.

McLaren, K.P., McDonald, M.A., 2005. Seasonal patterns of flowering and fruiting in a dry tropical forest in Jamaica. Biotropica 37, 584-590.

Miller-Rushing, A.J., Weltzin, J., 2009. Phenology as a tool to link ecology and sustainable decision making in a dynamic environment. New Phytol. 184, 743745.

Morellato, L.P., Talora, D.C., Takahasi, A., Bencke, C.C., Romera, E.C., Ziparro, V.B., 2000. Phenology of Atlantic Rain Forest trees: a comparative study. Biotropica 32, 811-823.

Morellato, L.P.C., Camargo, M.G.G., D’EcaNeves, F.F., Luize, B.G., Mantovani, A., Hudson, I.L., 2010. The influence of sampling method, sample size, and frequency of observations on plant phenological patterns and interpretation in tropical forest trees. In: Hudson, I.L., Keatley, M.R. (Eds.), Phenological research: methods for environmental and climate change analysis, doi:http:// dx.doi.org/10.1007/978-90-481-3335-2_5.

Morellato, L.P.C., Camargo, M.G.G., Gressler, E., 2013. A review of plant phenology in South and Central America. In: Schwartz, M.D. (Ed.), Phenology: An Integrative Environmental Science. Springer, Dordrecht, pp. 91-113.

Morellato, L.P., Alberton, B., Alvarado, S., Borges, B., Buisson, E., Camargo, M.G.G., Cancian, L., Carstensen, D.W., Escobar, D., Leite, P., Mendoza, I., Rocha, N., Soares, N., Silva, T., Staggemeier, V., Streher, A., Vargas, B., Peres, C., 2016. Linking plant phenology to conservation biology. Biol. Conserv. 195, 60-72.

Newstrom, L.E., Frankie, G.W., Baker, H.G., 1994. A new classification for plant phenology based on flowering patterns in lowland tropical rain forest trees at La Selva, Costa Rica. Biotropica 26, 141-159.

Primack, R.B., 1987. Relationships among flowers, fruits, and seeds. Ann. Rev. Ecol. Syst. $18,409-430$.

R Core Team, 2014. R: A Language and Environment for Statistical Computing. R Foundation for Statistical Computing, Vienna, Austria <http://www.R-project. org/> (accessed 24.09.16).

Rivera, G.S., Stephen, E., Caldas, L.S., Nicolassi, G., Coradin, R., Borchert, R., 2002. Increasing day length induces spring flushes of tropical dry forest trees in the absence of rain. Trees 16, 445-456.

Scheifinger, F., Templ, B., 2016. Is citizen science the recipe for the survival of paperbased Phenological Networks in Europe? BioScience 66, 533-534. 
SEMARNAT [Secretaría de Medio Ambiente y Recursos Naturales], 2010. Norma Oficial Mexicana NOM-059-SEMARNAT-2010. Diario Oficial de la Federación, December 30, 2010.

Singh, K.P., Kushwaha, C.P., 2005. Emerging paradigms of tree phenology in dry tropics. Curr. Sci. India 89, 964-975.

Singh, K.P., Kushwaha, C.P., 2006. Diversity of flowering and fruiting phenology of trees in a tropical deciduous forest in India. Ann. Bot. 97, 265-276.

Tjur, T., 2009. Coefficients of determination in logistic regression models - a new proposal: the coefficient of discrimination. Am. Stat. 63, 366-372.

Valdez-Hernández, M., Andrade, J.L., Jackson, P.C., Rebolledo-Vieyra, M., 2010. Phenology of five tree species of a tropical dry forest in Yucatán, México: effects of environmental and physiological factors. Plant Soil 329, 155-171. van Schaik, C.P., Terborgh, J.W., Wright, S.J., 1993. The phenology of tropical forest: significance and consequences for primary consumers. Ann. Rev. Ecol. Syst. 24 353-357.

Venter, S.M., Witkowski, E.T.F., 2011. Baobab (Adansonia digitata L.) fruit production in communal and conservation land-use type in Southern Africa. For. Ecol. Manage. 261, 630-639.

Wallace, R.B., Painter, R.L.E., 2002. Phenological patterns in southern Amazonian tropical forest: implications for sustainable management. For. Ecol. Manage. $160,19-33$.

Zar, J.H., 1999. Biostatistical Analysis. Prentice Hall, New Jersey. 